\title{
Simulation of collaborative product development knowledge diffusion using a new cellular automata approach
}

\author{
Kunpeng, Y. ${ }^{a}$, Jiafu, S. ${ }^{b,{ }^{*}}$, Hui, H. ${ }^{b}$ \\ ${ }^{\mathrm{a}}$ Managemengt College of Ocean University of China, Qingdao, P.R. China \\ ${ }^{b}$ Chongqing Technology and Business University, Chongqing Key Laboratory of Electronic Commerce \& Supply Chain System, \\ Chongqing, P.R. China
}

\begin{abstract}
A B S T R A C T
In order to quantitatively examine the diffusion process and pattern of collaborative product development (CPD), this paper puts forward a quantitative research model of CPD knowledge diffusion based on improved cellular automata. In light of the idea of SIS epidemic model and the local knowledge interaction characteristic of CPD knowledge diffusion, the influencing factors of knowledge diffusion are abstracted into the parametric variables in the process of knowledge diffusion, and the knowledge-SIS (K-SIS) model is constructed based on improved cellular automata for CPD knowledge diffusion. Finally, the K-SIS model is simulated to study the diffusion process and pattern of CPD knowledge, revealing the influence mechanism of CPD knowledge diffusion influencing factors on the diffusion process. The research results provide valuable reference for improving the efficiency of CPD knowledge diffusion.
\end{abstract}

\section{ARTICLE INFO}

Keywords:

Collaborative product development Knowledge diffusion Influencing factors

Cellular automata

*Corresponding author: jiafu.su@hotmail.com (Jiafu, S.)

Article history:

Received 19 June 2017

Revised 30 June 2017

Accepted 2 July 2017

(C) 2017 PEI, University of Maribor. All rights reserved.

\section{References}

[1] Yang, Y., Guo, B., Yin, S., Wang, W.-L., Zhang, X.-D. (2008). Connotation, theory framework and application of customer collaborative innovation, Computer Integrated Manufacturing Systems, Vol. 14, No 5, 944-950.

[2] Xing, L. (2016). Research on cooperation innovation among enterprises in the strategic emerging industrial cluster, Advances in Modelling and Analysis A, Vol. 53, No. 2, 93-107.

[3] Reagans, R., McEvily, B. (2003). Network structure and knowledge transfer: The effects of cohesion and range, Administrative Science Quarterly, Vol. 48, No. 2, 240-267, doi: 10.2307/3556658.

[4] Kim, H., Park, Y. (2009). Structural effects of R\&D collaboration network on knowledge diffusion performance, Expert Systems with Applications, Vol. 36, No. 5, 8986-8992, doi: 10.1016/j.eswa.2008.11.039.

[5] Li, L., Dang, X., Zhang, S. (2007). Study on the knowledge diffusion of enterprises technology innovation cooperation based on the perspective of knowledge potential, Science of Science and Management of S. \& T., Vol. 28, No. 4, 107-112, doi: 10.3969/j.issn.1002-0241.2007.04.024.

[5] Bass, F.M. (1969). A new product growth for model consumer durables, Management Science, Vol. 15, No. 5, 215227, doi: $10.1287 / \mathrm{mnsc} .15 .5 .215$.

[7] Sun, Y.-W., Wei, Y.-P. (2011). Empirical study on AIDA model of knowledge diffusion in high-tech enterprise alliance based on complex network, China Soft Science, Vol. 6, 17-26, doi: 10.3969/j.issn.1002-9753.2011.06.013.

[8] Meng, X., Liu, H., Wu, H. (2004). Multi-agent model of knowledge diffusion in network space, Studies in Science of Science, Vol. 21, No. 6, 636-641, https://doi.org/10.3969/j.issn.1003-2053.2003.06.014.

[9] Xue, J., Ding, C.Q., Yuan, J. (2016). The evolutionary game study of knowledge transfer behavior in cooperative crowdsourcing community of innovation, Advances in Modelling and Analysis A, Vol. 53, No. 2, 164-175.

[10] Yang, Y., Wang, X.-L., Zeng, Q., Yang, J., Xing, Q.-S. (2011). Multi-agent conflict coordination in collaborative product creative design optimization, Computer Integrated Manufacturing Systems, Vol. 17, No. 1, 1-9, doi: 10.13196/j.cims.2011.01.3.yangy.001. 
[11] Yang, J., Yang, Y., Zhao, C., Liang, X.-D., Zeng, Q. (2009). Integrated technology of customer knowledge in collaborative product innovation based on ontology theory, Computer Integrated Manufacturing Systems, Vol. 15, No. 12, 1-9, doi: 10.13196/j.cims.2009.12.17.yangj.024.

[12] Wolfram, S. (1984). Cellular automata as models of complexity, Nature, Vol. 311, No. 5985, 419-424, doi: $10.1038 / 311419 \mathrm{a} 0$.

[13] Guseo, R., Guidolin, M. (2009). Modelling a dynamic market potential: A class of automata networks for diffusion of innovations, Technological Forecasting and Social Change, Vol. 76, No. 6, 806-820, doi: 10.1016/i.techfore. 2008.10.005.

[14] Gu, B., Sheng, V.S. (2016). A robust regularization path algorithm for v-support vector classification, IEEE Transactions on Neural Networks and Learning Systems, Vol. 28, No. 5, 1241-1248, doi: 10.1109/TNNLS.2016.2527796.

[15] Sun, G.-Q., Jin, Z., Li, L. (2011). Emergent turing pattern in epidemic spreading using cellular automaton, International Journal of Modern Physics B, Vol. 25, No. 32, 4605-4613, doi: 10.1142/S0217979211059401.

[16] Xia, Z., Wang, X., Sun, X., Liu, Q., Xiong, N. (2016). Steganalysis of LSB matching using differences between nonadjacent pixels, Multimedia Tools and Applications, Vol. 75, No. 4, 1947-1962, doi: 10.1007/s11042-014-2381-8.

[17] Luo, S., Du, Y., Liu, P., Xuan, Z., Wang, Y. (2015). A study on coevolutionary dynamics of knowledge diffusion and social network structure, Expert Systems with Applications, Vol. 42, No. 7, 3619-3633 doi: 10.1016/j.eswa. $\underline{2014.12 .038 .}$ 


\section{APEM}

\title{
Simulacija difuzije znanja pri skupinskem razvoju izdelka s pomočjo novega pristopa celičnih avtomatov
}

\author{
Kunpeng, Y. ${ }^{a}$, Jiafu, S. $^{\text {b. }}{ }^{,}$, Hui, $\mathrm{H}^{\mathrm{b}}$ \\ ${ }^{a}$ Managemengt College of Ocean University of China, Qingdao, P.R. China \\ ${ }^{\mathrm{b}}$ Chongqing Technology and Business University, Chongqing Key Laboratory of Electronic Commerce \& Supply Chain System, \\ Chongqing, P.R. China
}

\begin{abstract}
POVZETEK
Da bi kvantitativno proučili difuzijski proces in vzorce pri skupinskem razvoju izdelkov (CPD), je v tem prispevku predstavljen kvantitativni model difuzije znanja pri CPD na podlagi metode izboljšanih celičnih avtomatov. Na podlagi SIS modela - in za difuzijo znanja pri CPD značilne interakcije lokalnega znanja - so vplivni dejavniki difuzije znanja povzeti v parametrične spremenljivke. Na podlagi izboljšanih celičnih avtomatov za difuzijo znanja CPD je bil zasnovan K-SIS (angl. knowledge-SIS) model. Za proučevanje difuzijskega procesa in vzorcev prehajanja znanja pri CPD je bila izvedena simulacija K-SIS modela. Ta je razkrila mehanizem dejavnikov, ki vplivajo na difuzijo $\mathrm{v}$ difuzijskem procesu pri CPD. Rezultati raziskave nudijo dragoceno referenco za izboljšanje učinkovitosti difuzije znanja pri CPD.

(C) 2017 PEI, University of Maribor. All rights reserved.
\end{abstract}

\section{PODATKI O ČLANKU}

Ključne besede:

Skupinski razvoj izdelka

Difuzija znanja

Vplivni dejavniki

Celični avtomati

*Kontaktna oseba: jiafu.su@hotmail.com

(Jiafu, S.)

Zgodovina članka:

Prejet 19. junija 2017

Popravljen 30. junija 2017

Sprejet 2. julija 2017 